\title{
Correspondence
}

\section{Detection of soluble BAG3 and anti-BAG3 antibodies in patients with chronic heart failure}

\author{
M De Marco ${ }^{1,2,10}$, A Falco ${ }^{1,2,10}$, A Basile ${ }^{1,2}$, A Rosati $^{1,2}$, M Festa $^{1,2}$, M d'Avenia $^{1,2}, M_{\text {Pascale }}^{1,2}, F_{\text {Dal Piaz }}{ }^{1}$, R Bisogni $^{2}$, D Barcaroli ${ }^{3}$, \\ G Coppola ${ }^{4}$, F Piscione ${ }^{5,6}$, A Gigantino ${ }^{5}$, R Citro ${ }^{5}$, R De Rosa ${ }^{6}$, G Vitulano ${ }^{5}$, N Virtuoso ${ }^{5}$, F Manganelli ${ }^{7}$, E Palermo ${ }^{7}$, F Siano $^{7}$, \\ G Rosato ${ }^{7}$, M Hahne ${ }^{8}$, C Tiberti ${ }^{9}$, V De Laurenzi ${ }^{2,3}$ and MC Turco ${ }^{\star, 1,2}$
}

Cell Death and Disease (2013) 4, e495; doi:10.1038/cddis.2013.8; published online 14 February 2013

Subject Category: Experimental Medicine

\section{Dear Editor,}

$\mathrm{Bcl}-2$-associated athanogene 3 (BAG3) protein belongs to the family of co-chaperones that interact with the ATPase domain of the heat shock protein HSP70 through the BAG domain (amino acids 110-124). BAG3 contains also a WW domain, a proline-rich repeat (PXXP) and two conserved Ile-Pro-Val motifs that mediate binding to other proteins. ${ }^{1}$ bag 3 gene is constitutively expressed in a few normal cell types, including myocytes, and in several primary tumors, while its expression is inducible by stressors in other cell types. Many evidence indicate that BAG3 has a role in sustaining cell survival, through mechanisms that, depending on the cell context, rely on the ability of BAG3 to modulate levels or localization of apoptosis-regulating proteins, such as IKK $\gamma$, Bax or BRAF, in either an Hsp70-dependent or -independent fashion. ${ }^{1-3}$

BAG3 is expressed during cardiomyoblast differentiation and sustains myogenin expression. In cardiomyocytes, BAG3 protein localizes at the Z-disc and interacts with the actin capping protein, CapZ $\beta 1$, stabilizing myofibril structure; BAG3 mutations can impair the Z-disc assembly and increase the sensitivity to stress-induced apoptosis. Mutations in bag3 gene have been associated with some forms of myofibrillar myopathy and dilated cardiomyopathy. ${ }^{4-7}$

Interestingly, we detected BAG3 protein in supernatants of the rat cardiomyocyte cell line $\mathrm{H} 9 \mathrm{c} 2$ and adult Human Cardiac Myocytes ( $\mathrm{HCMa}$ ) after $16 \mathrm{~h}$ of serum deprivation (Figure 1a). We then verified that BAG3 was present in extracellular vesicles isolated through a differential centrifugation procedure (Supplementary Figure S1A). To investigate the possibility that BAG3 could be released in vivo and become detectable in sera in conditions in which the heart is undergoing chronic stress, we analyzed sera from two patients affected by chronic heart failure (CHF). By western blot analysis, we detected a band recognized by anti-BAG3 antibody at the expected molecular weight in sera from patients but not from healthy donors (Figure 1b). We excised the band from a replica gel and subjected it to mass spectrometry, unmistakably identifying BAG3 (Supplementary Figure S1B). Furthermore, we found that sera from $\mathrm{CHF}$ patients recognized BAG3 protein in western blotting, using an anti-human IgG as secondary antibody (Supplementary Figure S1C). This result indicated the presence of anti-BAG3 antibodies in CHF patients' sera. To confirm this finding, we developed an ELISA test using recombinant BAG3 to coat plates and anti-human IgG to reveal and analyzed sera from $52 \mathrm{CHF}$ patients (EF $<45 \%$ ), compared with sera from 84 healthy donors. As shown in Figure 1c (and in Supplementary Figure S1D), we detected significantly higher values of anti-BAG3 antibodies in patients' compared with controls' sera. These data suggest that upon cardiac stress cardiomyocytes release BAG3 and this in turn results in production of auto-antibodies. There is no correlation with NYHA scores and antibody levels at this stage but screening of a larger number of patients in the future might be necessary to reveal potential correlations.

These results describe for the first time an extracellular BAG3 (eBAG3) released by stressed cardiomyocytes. As BAG3 lacks the consensus signal required for secretion via ER-Golgi pathway, it is likely to be released by the nonclassical secretory pathway. ${ }^{8}$ eBAG3 release by stressed cardiomyocytes appears to result in production of autoantibodies that could potentially be used as a biomarker for CHF patients, in combination with other already established markers. The presence of anti-BAG3 antibodies in $\mathrm{CHF}$ patients' sera indicates that released BAG3 can activate the immune system, and might therefore exert positive or negative functional effects on cardiac function, depending on the context. Future studies are required to clarify the biological roles of BAG3 and anti-BAG3 antibodies in $\mathrm{CHF}$,

\footnotetext{
${ }^{1}$ Department of Pharmaceutical and Biomedical Sciences, University of Salerno, Fisciano SA, Italy; ${ }^{2}$ BIOUNIVERSA s.r.l., University of Salerno, Fisciano SA, Italy; ${ }^{3}$ Department of Biomedical Sciences, University 'G. d'Annunzio' Chieti-Pescara, Fondazione 'G. D’Annunzio, Centro Studi sull'Invecchiamento, Ce.S.I.', Chieti, Italy; ${ }^{4}$ Unit of Transfusion Medicine, University Hospital 'San Giovanni di Dio e Ruggi d'Aragona', Salerno, Italy; 'Department of Cardiology, University Hospital 'San Giovanni di Dio e Ruggi d'Aragona', Salerno, Italy; ${ }^{6}$ Department of Clinical Medicine, Cardiovascular Sciences and Immunology, Federico II University, Naples, Italy; ${ }^{7}$ Department of Cardiology, 'A.O.S.G. Moscati' Hospital, Avellino, Italy; ${ }^{8}$ Institut de Génétique Moléculaire de Montpellier, CNRS UMR5535, Montpellier, France and ${ }^{9}$ Department of Clinical Sciences, University of Rome Sapienza, Rome, Italy

${ }^{*}$ Corresponding author: MC Turco, Department of Pharmaceutical and Biomedical Sciences, University of Salerno, Via Ponte don Melillo, 84084 Fisciano, Italy. Tel: +39089 969774; Fax: +39089 969602; E-mail: mcturco@unisa.it

${ }^{10}$ These authors contributed equally to this work.
} 
a

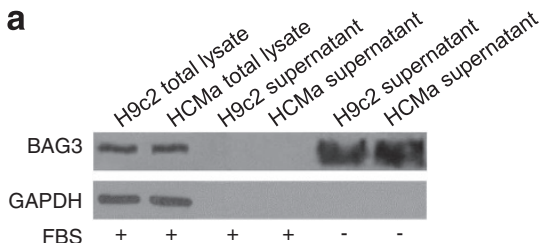

b

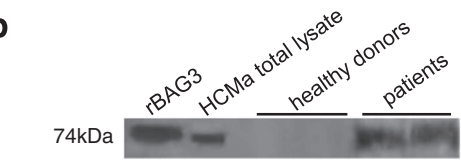

C

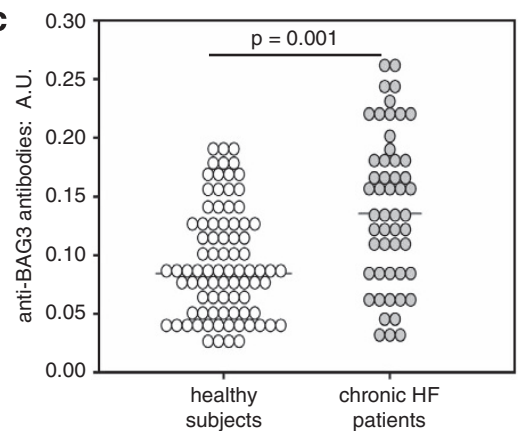

\begin{tabular}{|c|c|c|c|c|c|c|c|c|}
\hline & \multirow{2}{*}{$\begin{array}{l}\text { No. } \\
\text { Tot }\end{array}$} & \multirow{2}{*}{$\begin{array}{c}\text { age } \\
\text { average } \pm \\
\text { S.E. }\end{array}$} & \multicolumn{2}{|c|}{ Gender } & \multicolumn{4}{|c|}{ NYHA Class (No. Tot) } \\
\hline & & & M & $\mathrm{F}$ & 1 & II & III & IV \\
\hline $\begin{array}{l}\text { chronic HF } \\
\text { patients }\end{array}$ & 52 & $58.5 \pm 1.0$ & 43 & 9 & 30 & 9 & 10 & 3 \\
\hline $\begin{array}{l}\text { healthy } \\
\text { subjects }\end{array}$ & 84 & $49.6 \pm 0.7$ & 65 & 19 & & & & \\
\hline
\end{tabular}

and the utility of anti-BAG3 antibodies as a tool contributing to the study of the disease.

\section{Conflict of Interest}

MDM, AF, AB, MF, MDA, MP, VDL and MCT are shareholders of

BIOUNIVERSA s.r.I. that provided BAG3-specific antibodies and BAG3-

Figure 1 (a) Detection of BAG3 protein in supernatants from cultured cardiomyocytes. Human ( $\mathrm{HCMa}$ ) and rat ( $\mathrm{H} 9 \mathrm{c} 2)$ cardiomyocytes, at $80 \%$ confluence, were incubated with or without $10 \%$ FBS for $16 \mathrm{~h}$ at $37^{\circ} \mathrm{C}$ in a $5 \%$ $\mathrm{CO} 2$ atmosphere. Supernatants were dialyzed in a buffer containing $50 \mathrm{mmol} / \mathrm{I} \mathrm{NaCl}$ and $0.05 \%$ IGEPAL, lyophilized, resuspended in $1 \mathrm{ml}$ of RIPA buffer $(50 \mathrm{mmol} / \mathrm{T}$ Tris $\mathrm{HCl} \mathrm{pH} \mathrm{7.6,} 150 \mathrm{mmol} / \mathrm{I}$ sodium chloride, $2 \mathrm{mmol} / \mathrm{l}$ sodium orthovanadate, $4 \mathrm{mmol} / \mathrm{l}$ EDTA, $10 \mathrm{mmol} / /$ sodium pyrophosphate, $1 \% \mathrm{NP}-40,0.1 \%$ sodium deoxycholate) and analyzed with anti-BAG3 or anti-GAPDH antibodies by western blotting. (b) Mass analysis of BAG3 detected in the sera from two patients affected by CHF. The sera were analyzed with the anti-BAG3 polyclonal antibody TOS-2 in western blotting. (c) ELISA test for detection of anti-BAG3 antibodies in chronic HF patients. Serum samples from $52 \mathrm{CHF}$ patients $(\mathrm{EF}<45 \%)$ and from 84 healthy donors were analyzed for the presence of anti-BAG3 antibodies by ELISA. Results are plotted as arbitrary units (A.U.). Bars in the dot plot depict the median value obtained in the analyzed groups

specific ELISA tests free of charge for this work. All other authors have no industry relationship to disclose.

Acknowledgements. This work was supported by Ministero dell'Università (FARB) grants to MCT.

1. Rosati A et al. Cell Death Dis 2011; 2: e141.

2. Romano MF et al. Cell Death Differ 2003; 10: 383-385

3. Ammirante M et al. Proc Natl Acad Sci USA 2010; 107: 7497-7502.

4. Hishiya A, Kitazawa T, Takayama S. Circ Res 2010; 107: 1220-1231.

5. Arimura T et al. Hum Mutat 2011; 32: 1481-1491.

6. De Marco M, Turco MC, Rosati A. Cell Cycle 2011; 10: 850-852.

7. Labeit S. Hum Mutat 2011; 32: iv.

8. Doroudgar S et al. Trends Mol Med 2011; 17: 207-214.

(c) SOMERIGHTS RESERV licensed under the Creative Commons Attribution-NonCommercial-No Derivative Works 3.0 Unported License. To view a copy of this license, visit http://creativecommons.org/licenses/by-nc-nd/3.0/

Supplementary Information accompanies the paper on Cell Death and Disease website (http://www.nature.com/cddis) 